Objectives: To evaluate a triage strategy that offers the possibility to see patients within 4 weeks for short term appointments in order to check the probability of an inflammatory RMD and the necessity to further evaluate the patients in due time. Methods: Physician's and patient's information who called our tertiary rheumatology

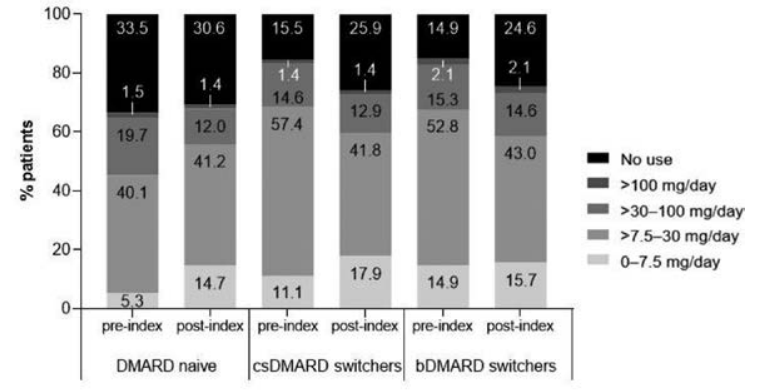

bDMARD, biologic disease-modifying antirheumatic drug; cSDMARD, conventional synthetic disease-modifying antirheumatic drug; DMARD, disease-modifying antirheumatic drug.

Figure 2. Pre-index and post-index glucocorticoid use by cohort

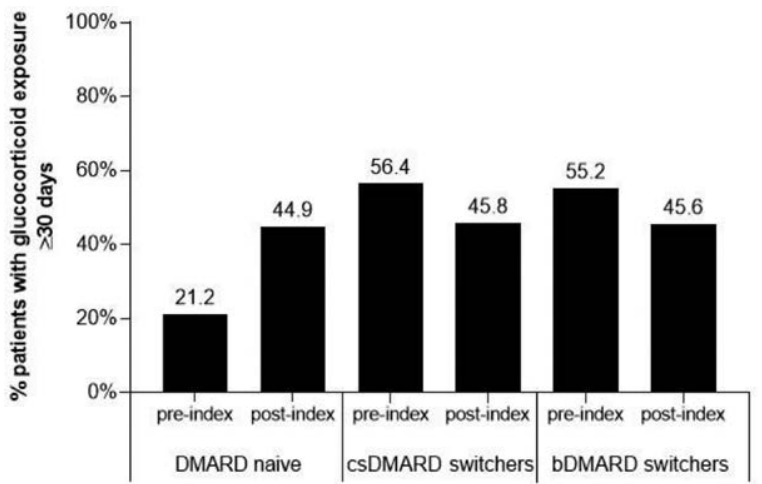

bDMARD, biologic disease-modifying antirheumatic drug; csDMARD, conventional synthetic disease-modifying antirheumatic drug; DMARD, disease-modifying antirheumatic drug.

Conclusion: Real world glucocorticoid use was high in all categories of DMARD-treated RA patients, at baseline and during their next treatment, suggesting ongoing medical needs. Glucocorticoid doses exceeded $7.5 \mathrm{mg} /$ day for most patients. In addition, many patients had $\geq 30$ days exposure to glucocorticoids, posing an additional safety risk.

Disclosure of Interests: Robin K Dore Grant/research support from: AbbVie, Amgen, Biogen, Bristol-Myers Squibb, Eli Lilly and Co., Gilead Sciences, Inc., GlaxoSmithKline, Myriad, Novartis, Pfizer, Radius, Regeneron, Sanofi, and UCB. Consultant of: AbbVie, Amgen, Biogen, Bristol-Myers Squibb, Eli Lilly and Co., Gilead Sciences, Inc., GlaxoSmithKline, Myriad, Novartis, Pfizer, Radius, Regeneron, Sanofi, and UCB., Jenya Antonova Employee of: Gilead Sciences. Inc. Magdaliz Gorritz Consultant of: Gilead Sciences, Inc., Lawrence Chang Consultant of: Gilead Sciences, Inc., Handing Xie Consultant of: Gilead Sciences, Inc. Mark C. Genovese Grant/research support from: Abbvie, Eli Lilly and Company, EMD Merck Serono, Galapagos, Genentech/Roche, Gilead Sciences, Inc., GSK, Novartis, Pfizer Inc., RPharm, Sanofi Genzyme, Consultant of: Abbvie, Eli Lilly and Company, EMD Merck Serono, Genentech/Roche, Gilead Sciences, Inc., GSK, Novartis, RPharm, Sanofi Genzyme

DOI: 10.1136/annrheumdis-2020-eular.3776

\section{AB1144 DAILY CLINICAL CARE OF PATIENTS WITH MUSCULOSKELETAL COMPLAINTS - HOW HELPFUL IS A TRIAGE SYSTEM FOR EARLY RECOGNITION OF INFLAMMATORY RHEUMATIC DISEASES?}

X. Baraliakos ${ }^{1}$, I. Redeker ${ }^{2}$, M. Zacharopoulou ${ }^{1}$, S. Tsiami ${ }^{1}$, K. Tsiaousi ${ }^{1}$, D. Morzeck', J. Braun ${ }^{1} .{ }^{1}$ Rheumazentrum Ruhrgebiet Herne, Ruhr-University Bochum, Herne, Germany; ${ }^{2}$ German Rheumatism research center, Berlin, Germany

Background: Early diagnosis and treatment are important for the management of inflammatory rheumatic diseases (RMD). However, the availability of rheumatologists is limited in most European countries and selection strategies lack sensitivity and/or specificity. department's outpatient clinic for a date in the triage system were included in this analysis. The time to first appointment as assessed by a nurse (Step 1), the short evaluation by a rheumatologist in the triage (Step 2) and the patient's complaints and the diagnoses after an extensive diagnostic evaluation (Step 3) were documented. Results: In a period of 9 months in 2018, a total of 982 patients presented. A total of 62 patients $(6.3 \%)$ were considered urgent (appointment within 3 days), while 240 (24.4\%) were appointed within 4 weeks at Step 2. Of the former $46(19.2 \%)$, and of the latter 151 patients $(62.9 \%)$ were diagnosed with inflammatory RMD at Step 3. In total, 334 patients (34.0\%) were diagnosed with inflammatory RMD at Step 3 including 126 with RA (37.7\%), 71 with axSpA/PsA (21.3\%), 95 with connective tissue disease/vasculitis (28.4\%) and 20 with gout $(6.0 \%)$. The diagnosis suspected in Step 2 was confirmed in Step 3 in $77.9 \%$ of cases. In 217 patients, the diagnosis suspected in Step 2 was not confirmed in Step 3. Of them, $34(15.7 \%)$ had unclear findings at Step 2 but an inflammatory RMD was found at Step 3, while 148 (68.2\%) had a suspected inflammatory RMD at Step 2 but this was not confirmed at Step 3. The most frequent musculoskeletal complaint at the time point of referral was pain in small peripheral joints (hands and/or feet) in 858 patients $(87.4 \%)$, in large peripheral joints (knees, shoulders and/or hips) in 780 patients $(79.4 \%)$ and back pain in 682 patients $(69.5 \%)$. Fever, night sweats and unclear weight loss was reported by 50 patients $(5.1 \%)$, while 210 patients $(24.5 \%)$ presented with findings suspicious of inflammatory RMD such as elevated CRP of unclear origin, and 43 patients $(4.8 \%)$ because of a threat of organ damage such as unclear elevation of creatinine, as reported by the referring physician. In addition 167 patients (17.0\%) had received glucocorticoids prior to referral, $87(52.1 \%)$ of which finally did not receive the diagnosis of inflammatory RMD at Step 3, while 737 patients $(75.1 \%)$ were receiving NSAIDs prior to referral.

Conclusion: In this prospective evaluation of a triage system where all patients were pre-screened by a nurse and were seen within 4 weeks by a rheumatologist, clinical differentiation could be performed timely due to a successfully structured triage system. The initially suspected diagnosis was finally confirmed in $\geq 75 \%$ of cases, while $\geq 1 / 3$ of patients had a definite inflammatory RMD.

This work was supported by an unrestricted Grant from Abbvie

Disclosure of Interests: Xenofon Baraliakos Grant/research support from: Grant/research support from: AbbVie, BMS, Celgene, Chugai, Merck, Novartis Pfizer, UCB and Werfen, Consultant of: AbbVie, BMS, Celgene, Chugai, Merck, Novartis, Pfizer, UCB and Werfen, Speakers bureau: AbbVie, BMS, Celgene Chugai, Merck, Novartis, Pfizer, UCB and Werfen, Imke Redeker: None declared, Maria Zacharopoulou: None declared, Styliani Tsiami: None declared, Konstantia Tsiaousi: None declared, Doris Morzeck: None declared, Juergen Braun Grant/research support from: Abbvie (Abbott), Amgen, BMS, Boehringer, Celgene, Celltrion, Centocor, Chugai, Eli Lilly and Company, Medac, MSD (Schering Plough), Mundipharma, Novartis, Pfizer (Wyeth), Roche, Sanofi- Aventis, and UCB Pharma, Consultant of: Abbvie (Abbott), Amgen, BMS, Boehringer Celgene, Celltrion, Centocor, Chugai, EBEWE Pharma, Eli Lilly and Company, Medac, MSD (Schering-Plough), Mundipharma, Novartis, Pfizer (Wyeth), Roche Sanofi-Aventis, and UCB Pharma, Speakers bureau: Abbvie (Abbott), Amgen, BMS, Boehringer, Celgene, Celltrion, Centocor, Chugai, EBEWE Pharma, El Lilly and Company, Medac, MSD (Schering-Plough), Mundipharma, Novartis, Pfizer (Wyeth), Roche, Sanofi-Aventis, and UCB Pharma DOI: 10.1136/annrheumdis-2020-eular.6529

\section{AB1145 2 PRESCRIPTION PATTERNS AND DISEASE ACTIVITY IN PORTUGUESE WOMEN OF CHILDBEARING AGE WITH RHEUMATOID ARTHRITIS, PSORIATIC ARTHRITIS, ANKYLOSING SPONDYLITIS AND JUVENILE IDIOPATHIC ARTHRITIS}

S. C. Barreira ${ }^{1,2}$, A. R. Cruz-Machado ${ }^{1,2}$, A. L. Fernandes ${ }^{3}$, I. Genrinho ${ }^{4}$, G. Sequeira ${ }^{3}$, P. Monteiro ${ }^{4}$, J. E. Fonseca ${ }^{1,2} .{ }^{1}$ Hospital de Santa Maria, Centro Hospitalar e Universitário de Lisboa Norte, Serviço de Reumatologia e Doenças Ósseas Metabólicas, Lisboa, Portugal; ${ }^{2}$ Instituto de Medicina Molecular, Faculdade de Medicina, Universidade de Lisboa, Centro Académico de Medicina de Lisboa, Portugal, Unidade de Investigação em Reumatologia, Lisboa, Portugal; ${ }^{3}$ Centro Hospitalar Universitário do Algarve Serviço de Reumatologia, Faro, Portugal; ${ }^{4}$ Centro Hospitalar Tondela-Viseu, Unidade de Reumatologia, Viseu, Portugal

Background: Disease activity (DA) at conception is one of the main predictors of pregnancy outcomes in women of childbearing age (WoCBA) with rheumatic diseases. Teratogenicity and unawareness about pregnancy compatibility of some disease-modifying anti-rheumatic drugs might limit the choice of treatment in WoCBA Objectives: To assess differences in prescription patterns between WoCBA with rheumatoid arthritis (RA), psoriatic arthritis (PsA), ankylosing spondylitis (AS) and juvenile idiopathic arthritis (JIA) and comparator groups, namely 
postmenopausal women (PMW) and age-matched men. Evaluate DA in WoCBA comparing to the aforementioned groups.

Methods: Observational transversal study, using data from the portuguese registry of rheumatic diseases (Reuma.pt) from 3 portuguese centers. Adult patients (pts) with the diagnosis of RA, PsA, AS or JIA were allocated to the following groups: WoCBA (aged 18-44y), young men (YM) (18-44y), PMW ( $\geq 45 y$ ) and matched men $(\geq 45 y)$. Demographic and clinical variables are described as means or frequencies. Differences between groups regarding therapy and DA were assessed with Chi-square and ANOVA tests. Linear and logistic regression models were used to find predictors of DA and prescription patterns.

Results: 2133 pts were included, $69.9 \%$ female with a mean age of $55.96 \pm 15.85$ y. 1437 pts were diagnosed with RA, 305 with PsA, 254 with AS and 137 with JIA. Patterns of prescription are detailed in table 1. WoCBA were less likely to be treated with glucocorticoids than PMW (OR 0.66 95\% Cl 0.44-0.99). WoCBA were 1.76 times more likely to be treated with MTX than YM $(95 \% \mathrm{Cl} 1.04-2.97)$. Certolizumab was specially prescribed in WoCBA (OR 13.8, 95\%Cl 1.4-132.8). WoCBA had significantly higher DA scores than YM (DAS28 $3.03 \pm 1.39$ vs $2.32 \pm 1.18$ and BASDAI $3.55 \pm 2.0$ vs $2.43 \pm 1.66$ )

Table 1. Prescription patterns

\begin{tabular}{lccccc}
\hline Medications, n (\%) & $\begin{array}{c}\mathrm{A} \text {-WoCBA } \\
(\mathrm{N}=256)\end{array}$ & $\begin{array}{c}\mathrm{B} \text { - Young } \\
\text { Men } \\
(\mathrm{N}=161)\end{array}$ & $\begin{array}{c}\mathrm{C} \text { - Post menopau- } \\
\text { sal Women } \\
(\mathrm{N}=927)\end{array}$ & $\begin{array}{c}\mathrm{D}-\text { Men } \\
\mathrm{N}=340\end{array}$ & $\begin{array}{c}\text { Chi-square } \\
\text { test }\end{array}$ \\
\hline NSAIDs & $143(55.9)$ & $111(68.9)$ & $472(50.9)$ & $169(49.7)$ & $\mathrm{p}<0.001$ \\
Glucocorticoids & $106(41.4)$ & $31(19.3)$ & $625(67.4)$ & $154(45.3)$ & $\mathrm{p}<0.001$ \\
csDMARDs & & & & & \\
- Methotrexate & $149(58.2)$ & $60(37.3)$ & $663(71.5)$ & $197(57.9)$ & $\mathrm{p}<0.001$ \\
- Leflunomide & $12(4.7)$ & $4(2.5)$ & $45(4.9)$ & $2(0.6)$ & $\mathrm{p}=0.003$ \\
- Sulfassalazine & $9(3.5)$ & $5(3.1)$ & $39(4.2)$ & $9(2.7)$ & $\mathrm{NS}$ \\
- Hydroxychloroquine & $36(14.1)$ & $4(2.5)$ & $117(12.6)$ & $18(5.3)$ & $\mathrm{p}<0.001$ \\
bDMARDs & & & & & \\
- Etanercept & $48(18.8)$ & $29(18.0)$ & $140(15.1)$ & $66(19.4)$ & $\mathrm{NS}$ \\
- Infliximab & $9(3.5)$ & $11(6.8)$ & $36(3.9)$ & $30(8.8)$ & $\mathrm{p}=0.002$ \\
- Adalimumab & $17(6.6)$ & $15(9.3)$ & $47(5.1)$ & $27(7.9)$ & $\mathrm{NS}$ \\
- Golimumab & $15(5.9)$ & $18(11.2)$ & $37(4.0)$ & $30(8.8)$ & $\mathrm{p}<0.001$ \\
- Certolizumab & $10(3.9)$ & $0(0)$ & $2(0.3)$ & $2(0.6)$ & $\mathrm{p}<0.001$ \\
- Tocilizumab & $12(4.7)$ & $2(1.2)$ & $71(7.7)$ & $10(2.9)$ & $\mathrm{p}<0.001$ \\
- Rituximab & $5(1.9)$ & $0(0)$ & $50(5.4)$ & $4(1.2)$ & $\mathrm{p}<0.001$ \\
- Abatacept & $0(0)$ & $0(0)$ & $9(1)$ & $0(0)$ & $\mathrm{NS}$ \\
- Secukinumab & $1(0.4)$ & $4(3.5)$ & $8(0.9)$ & $2(0.6)$ & $\mathrm{NS}$ \\
- Ustekinumab & $1(0.4)$ & $3(1.9)$ & $5(0.5)$ & $0(0)$ & $\mathrm{NS}$ \\
tsDMARDs & $4(1.6)$ & $1(0.6)$ & $9(0.9)$ & $0(0)$ & $\mathrm{NS}$ \\
\hline
\end{tabular}

bDMARDs - biologic disease modifying antirheumatic drugs, csDMARDs - conventional synthetic disease modifying antirheumatic drugs, NSAIDs - non-steroidal anti-inflammatory drugs, tsDMARD - targeted synthetic disease modifying antirheumatic drugs, WoCBA women of childbearing age

Conclusion: Certolizumab was prescribed preferentially in WoCBA, who alsoreceived more MTX than YM. Nevertheless, DA in this group was not well controlled, which may influence future pregnancy outcomes. Ensuring tight DA control in WoCBA through proper and ideally no teratogenic medication remains an unmet clinical need.

Disclosure of Interests: None declared

DOI: 10.1136/annrheumdis-2020-eular.1976

\section{AB1146 REAL-LIFE PRACTICES IN MANAGEMENT OF REPRODUCTIVE HEALTH IN SLE AND APS BY OBSTETRICIANS AND RHEUMATOLOGISTS IN EGYPT. (AN ONLINE-BASED QUESTIONNAIRE)}

A. Beltagy ${ }^{1}$, N. Eshak ${ }^{2}$, M. Morsy ${ }^{1}$, S. Shoela ${ }^{3}$, F. Fayed ${ }^{4}$, S. Aly ${ }^{5}$, A. Emam ${ }^{6}$, A. El-Girby ${ }^{7} .^{1}$ Alexandria University, Faculty of Medicine, Internal Medicine, Rheumatology Department, Alexandria, Egypt; ${ }^{2}$ Texas Tech University Health Sciences Center, Internal medicine, Lubbock, United States of America; ${ }^{3}$ Alexandria University, Faculty of Medicine, Internal Medicine, Rheumatology Department, Alexandria, Egypt; ${ }^{4}$ Alexandria University, Alexandria Students Hospital, Internal Medicine, Rheumatology, Alexandria, Egypt; ${ }^{5}$ Alexandria University, Alexandria, Egypt; ${ }^{6}$ Alexandria University, Obstetric and Gynecology, Alexandria, Egypt; ${ }^{7}$ Alexandria University, Internal Medicine, Rheumatology, Alexandria, Egypt

Background: Systemic lupus erythematosus (SLE) is an auto-immune disease that affect women in their reproductive age. Antiphospholipid syndrome (APS) is a hypercoagulable immune disease that occur as a primary condition or in assosiation with SLE.

The reproductive aspects as contraception, fertility, pregnancy are crucial to consider for proper management of SLE/APS.
Addressing these issues require collaboration between rheumatologists and obstetricians, improving their knowledge and ensuring that both are acquainted with the updated guidelines.

Objectives: To assess the knowledge and practice of Egyptian obstetricians and rheumatologists in management of reproductive health issues in SLE and APS, and to detect common misconceptions.

Methods: This research was conducted via google form online survey based on points discussed in EULAR recommendations for women's health and the management of family planning, assisted reproduction, pregnancy and menopause in patients with SLE and/or APS. ${ }^{1}$ It was sent to target obstetricians and rheumatologists by internet clouds like (Facebook, twitter, Linkedln) from August to November 2019. It included five domains; demographic data, general knowledge and attitudes about pregnancy in SLE and APS, contraception, drugs, and assisted reproductive techniques (ART)

After submitting answers, respondents were shown a link directing them to the 2016 EULAR recommendations. ${ }^{1}$

Results: This study was conducted on 254 physicians, $62 \%$ obstetricians and $38 \%$ rheumatologists. $64.6 \%$ were between the ages of $30-35$ years.

For general knowledge, $52 \%$ of Obstetricians considered pregnancy in inactive SLE to be risky. ( $79.4 \%$ vs $54.1 \%$ ) of (rheumatologists and obstetricians) respectively test for aPL in SLE patients. More than $70 \%$ in both groups were well informed on the increased rate of fetal and maternal complications in both SLE and APS.

For fetal surveillance, $87 \%$ and $90 \%$ of obstetricians preformed first and second trimester U/S, and $79 \%$ preformed second trimester Doppler.

For contraception, $(57.7 \%$ vs $56.7 \%$ ) discuss contraceptive choices with their patients. The majority considered it safe to use IUDs $(73.9 \%$ vs $76 \%)$ and condoms ( 84.7 vs $85.4 \%$ ) in both SLE and APS patients. On the other hand, for hormonal contraception- Levonorgestrol IUD, Depoprovera, COCP, and POP- only $14.6 \%, 22.9 \%, 26.1 \%, 24.8 \%$ of rheumatologists and $18.5 \%, 27.2 \%, 29.9 \%, 26.8 \%$ of obstetricians considered them unsafe to use in APS.

Concerning treatment, the majority considered low dose presnidone to be safe during pregnancy $(94.8 \%$ vs $80 \%)$ and breastfeeding $(87.6 \%$ vs $64.3 \%)$. The majority also agreed on avoidance of Methotrexate $(94.8 \%$ vs $84.1 \%)$ and Cyclophosphamide $(89.7 \%$ vs $66.2 \%)$. However, regarding Hydroxychloroquine and Azathioprine use in pregnancy there was a significant discrepancy between rheumatologists and obstetricians, $(89.7 \%$ vs $42 \%)$ and $(78.4 \%$ vs $36.9 \%)$ believed them safe to use in pregnancy. For Mycophenolate Mofetil, $(80.4 \%$ vs $46.5 \%)$ said that it should be avoided in pregnancy. Regarding ART $(45.4 \%$ vs $71 \%$ ) considered it safe to use in stable SLE/APS

Conclusion: The gaps in knowledge identified include the use of hormonal contraception in APS patients and the proper utilization of important medications to prevent and treat lupus flares. Initiation of shared Rheumatology/ obstetric clinics and focusing on the identified educational topics, would lessen the gap in knowledge and discrepancies in practice improve overall patient management. References:

[1] Andreoli L et al. Ann Rheum Dis. 2017;76(3):476-85.

Disclosure of Interests: None declared

DOI: 10.1136/annrheumdis-2020-eular.2162

\section{AB1147 SCREENED - HIGH REMISSION RATES UNDERLINE THE BENEFIT OF SCREENING CONSULTATION MODELS FOR EARLY RECOGNITION AND TREATMENT OF RHEUMATIC AND MUSCULOSKELETAL DISEASES}

K. Benesova ${ }^{1}$, V. Lion ${ }^{1}$, O. Hansen ${ }^{1}$, H. M. Lorenz ${ }^{1}{ }^{1}$ University Hospital Heidelberg, Department of Medicine V, Hematology, Oncology and Rheumatology, Heidelberg, Germany

Background: Early recognition and treatment of rheumatic and musculoskeletal diseases (RMDs) is of critical importance for the individual outcome. However, nationwide health care structures in Germany do not facilitate early access to initial rheumatologic evaluation. Furthermore, waiting times of several months due to substantial capacity constrains in regional rheumatology care services compromise the prognostically relevant "window of opportunity" for subsequent sustained remission. To promote early detection of RMDs, the Division of Rheumatology at the University hospital Heidelberg, Germany has launched a unique screening consultation model that offers early access to rheumatologic evaluation on regional level.

Objectives: The registry-based study SCREENED ("Screen for early diagnosis") has been initiated to monitor the outcome of patients that were diagnosed with an RMD at the screening clinic and to assess the costs and benefits of this consultation model for the regional quality of care. 